\title{
Selective autophagy
}

\section{Mohammad Omar Faruk $^{1,2}$ (D) | Yoshinobu Ichimura ${ }^{1}$ (D) | Masaaki Komatsu ${ }^{1}$ (ID}

\author{
${ }^{1}$ Department of Physiology, Juntendo \\ University Graduate School of Medicine, \\ Bunkyo-ku, Japan \\ ${ }^{2}$ Department of Cell Physiology, Niigata \\ University Graduate School of Medical and \\ Dental Sciences, Chuo-ku, Japan

\section{Correspondence} \\ Masaaki Komatsu, Hongo 2-1-1 Bunkyo-ku, \\ Tokyo 113-8421, Japan. \\ Email: mkomatsu@juntendo.ac.jp \\ Funding information \\ Japan Society for the Promotion of \\ Science, Grant/Award Number: $21 \mathrm{H} 00477$, \\ $20 \mathrm{K06644}$ and 19H05706; Ministry of \\ Education, Culture, Sports, Science and \\ Technology; Takeda Science Foundation
}

\begin{abstract}
While starvation-induced autophagy is thought to randomly degrade cellular components, under certain circumstances autophagy selectively recognizes, sequesters, and degrades specific targets via autophagosomes. This process is called selective autophagy, and it contributes to cellular homeostasis by degrading specific soluble proteins, supramolecular complexes, liquid-liquid phase-separated droplets, abnormal or excess organelles, and pathogenic invasive bacteria. This means that autophagy, like the ubiquitin-proteasome system, strictly regulates diverse cellular functions through its selectivity. In this short review, we focus on the mechanism of "selective" autophagy, which is rapidly being elucidated.
\end{abstract}

\section{KEYWORDS}

ATG8-family proteins, autophagy, liquid-liquid phase separation, selective autophagy, selective autophagy receptors

\section{1 | INTRODUCTION}

Since its discovery, autophagy has been regarded as the ultimate survival strategy of eukaryotes, as it is a "non-selective" self-degradation system that is markedly induced in response to nutrient starvation and that provides amino acids and other building blocks by degrading the organism's own cellular components. However, since the mechanism of selective degradation of ubiquitinated proteins by autophagy was proposed in 2005 by Terje Johansen's group, ${ }^{1}$ the selectivity of autophagy has attracted much attention, together with that of the molecular mechanism of the yeast Cvt pathway (a vacuolar enzyme transport pathway using the autophagy molecular mechanism) ${ }^{2}$ and mitochondrial selective degradation (mitophagy). ${ }^{3}$ To facilitate the understanding of selective autophagy, we would first like to briefly describe the basic mechanism of autophagosome formation. ${ }^{4}$ Autophagy is a system in which an isolation membrane/phagophore that forms in the vicinity of the endoplasmic reticulum (ER) is elongated, and a portion of its cytoplasm is sequestered into autophagosomes, which are then transported to lysosomes for degradation.
The proteins involved in the formation of autophagosomes are called "core ATG proteins," each of which consists of 6 functional units (Figure 1): (1) the ULK1 protein kinase complex, (2) ATG9 vesicles, (3) the phosphatidylinositol 3-kinase complex I (PI3K complex I), (4) the ATG2-WIPI complex, (5) the ATG12-ATG5-ATG16L complex, and (6) the ATG8 family protein-phosphatidylethanolamine (PE) conjugation system. When autophagy is induced, these functional units assemble at the site of autophagosome formation and form the preautophagosomal structure, also known as the phagophore assembly site (PAS). The formation of the PAS occurs in the vicinity of the vacuole and ER in budding yeast, and on the ER in mammals, especially at the contact site with mitochondria. The sequential action of these 6 functional units is responsible for the formation of the isolation membrane/phagophore (Figure 1). When the ULK1 protein kinase complex (1) is translocated to the ER subdomain (especially the mitochondrial contact site), PI3K complex I (3) is recruited and $\mathrm{PI}(3) \mathrm{P}$ production increases. WIPI, a $\mathrm{PI}(3) \mathrm{P}$-binding protein, binds to the $\mathrm{PI}(3) \mathrm{P}$ produced by $\mathrm{PI} 3 \mathrm{~K}$ complex I and accumulates with its binding partner ATG2 at the site of autophagosome formation (4). 
(5)

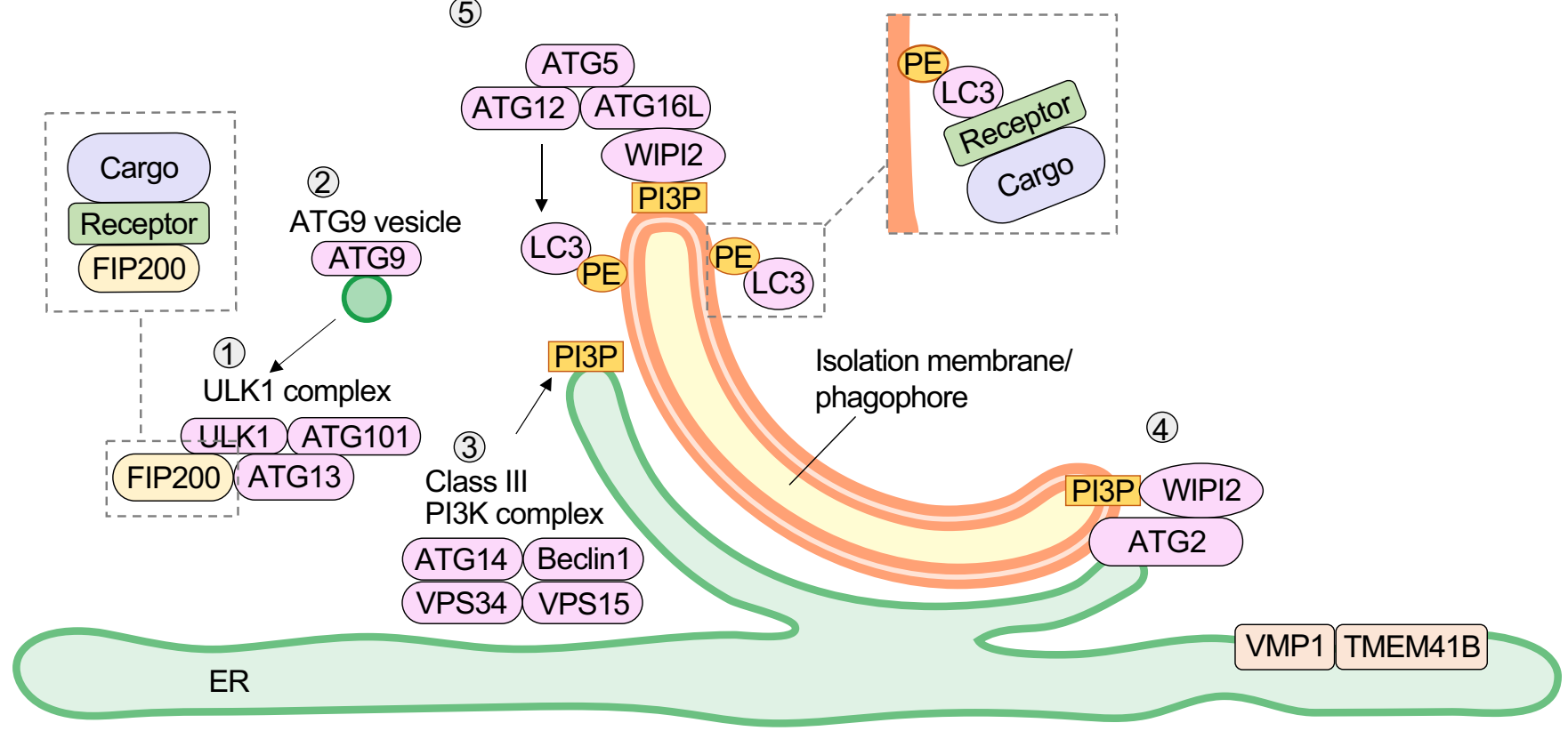

FIGURE 1 Autophagosome formation. When the ULK1 protein kinase complex1 is translocated to the ER subdomain, PI3K complex I (3) is recruited and the $\mathrm{PI}(3) \mathrm{P}$ production increases. WIPI binds to PI(3)P and accumulates with its binding partner, ATG2 (4). ATG2 connects the ER to the isolation membrane/phagophore and transports lipids. ATG9, a membrane protein (2), transiently accumulates in the isolation membrane/phagophore and scrambles phospholipids transported by ATG2 from the ER to the cytoplasmic layer of the isolation membrane/ phagophore. VMP1 and TMEM41B are ER membrane proteins that have been shown to be involved in regulating the cytoplasmic side of the ER. ATG12 and ATG5 are covalently bound to each other via a ubiquitin-like conjugation reaction. The ATG12-ATG5 conjugate forms a complex with ATG16L and localizes to the isolation membrane/phagophore (5), which determines the location of amide bond formation between ATG8 family proteins and PE. For selective autophagy, receptors that bind to both cargo and ATG proteins ensure selectivity. FIP200, a component of the ULK1 protein kinase complex and ATG9, interacts with these receptors to promote the formation of an isolation membrane/phagophore around the cargo. Interaction of these receptors with ATG8 family-PE results in the elongation of the isolation membrane/phagophore along the substrate

ATG2 connects the ER to the isolation membrane/phagophore and transports lipids. ${ }^{5}$ ATG9, a membrane protein, localizes to vesicles formed from the Golgi apparatus (2) and transiently accumulates in the isolation membrane/phagophore. ATG9 scrambles phospholipids transported by ATG2 from the ER to the cytoplasmic layer of the isolation membrane/phagophore, ${ }^{6,7}$ distributing them to both sides of the lipid bilayer and allowing the isolation membrane/phagophore to expand. The ER membrane proteins VMP1 and TMEM41B are responsible for scrambling phospholipids in the ER membrane and for regulating the contact between the ER and the isolation membrane/ phagophore. ${ }^{8,9}$ In the ATG12-conjugation system, ATG12 and ATG5 are covalently bound via a ubiquitin-like conjugation reaction. The ATG12-ATG5 conjugate forms a complex with ATG16L and localizes to the isolation membrane/phagophore (5), which determines the location of amide bond formation between ATG8 family proteins and PE. Autophagosomes surround a specific substrate, known as the cargo, and fuse with lysosomes to specifically degrade just the cargo, a process called selective autophagy. ${ }^{10}$ Depending on the type of cargo, the process is referred to as aggrephagy (cargo: protein aggregates), mitophagy (mitochondria), ER-phagy (ER), lysophagy (lysosomes), nucleophagy (nuclear membrane), xenophagy (invasive bacteria themselves or endosomes surrounding bacteria), pexophagy (peroxisomes), lipophagy (lipid droplets), or glycophagy (glycogen granules). ${ }^{10}$ For selective autophagy, FIP200, a component of the ULK1 protein kinase complex and ATG9, interacts with the receptor such as p62, Tax1BP1 and optineurin (see the next section for more information) to promote the formation of an isolation membrane/phagophore around the cargo. ${ }^{11-14}$ Interaction of the receptor with ATG8 family-PE results in the elongation of the isolation membrane/phagophore along the substrate. ${ }^{15,16}$

\section{2 | MOLECULAR MACHINERY OF SELECTIVE AUTOPHAGY}

\section{1 | Selective autophagy receptors}

The molecular mechanism of autophagosome formation is thought to be fundamentally the same in non-selective and selective autophagy. However, in selective autophagy, selectivity is ensured by specific labeling of each cargo depending on the stress and receptors (or adaptors). ${ }^{10,17}$ The phrase labeling of each cargo refers to the ubiquitination of the cargo and the localization of the receptor to the cargo. Conversely, receptors refer to a group of proteins that bind to both the cargo and to ATG proteins. Receptors can be divided into 2 types: ubiquitin-binding receptors, which recognize 
the ubiquitin chains of the cargo, and cargo-localizing receptors, which localize directly on the degraded cargo (Figure 2A). Cargolocalizing receptors are mainly used for selective autophagy in budding yeast, while both types of receptors are present in higher organisms. Selective autophagy by cargo-localizing receptors is regulated by the receptors' expression and localization. This is true in mitophagy and ER-phagy, and in the latter case several of these receptors are differentially localized in sheet-like or tubular ER and differentially induced by starvation, ER stress, etc. ${ }^{18,19}$ There are also several cargo-localizing receptors in mitophagy, and their expression is differentially regulated by starvation, hypoxia, and so on. ${ }^{20}$ Conversely, for selective autophagy using ubiquitin-binding receptors, ubiquitination of substrates by ubiquitin ligases is the major regulatory mechanism. Ubiquitination of mitochondrial outer membrane proteins by Parkin-PINK1 is the most well-characterized form of ubiquitination in depolarized mitochondria. ${ }^{21,22}$ In lysophagy, several ubiquitin ligases such as TRIM16, ${ }^{23} \mathrm{SCF}^{\mathrm{FBXO} 27},{ }^{24}$ and UBE2QL1 ${ }^{25}$ ubiquitinate lysosomal or endosomal membrane proteins, and in xenophagy, RNF $213^{26}$ and LUBAC ${ }^{27,28}$ recruit receptors by directly ubiquitinating bacteria.

(A)

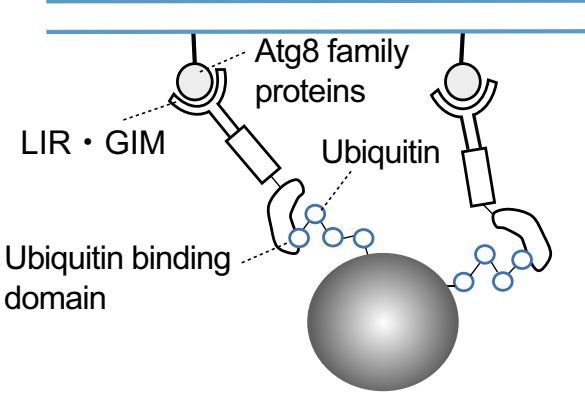

Ubiquitin-binding receptors

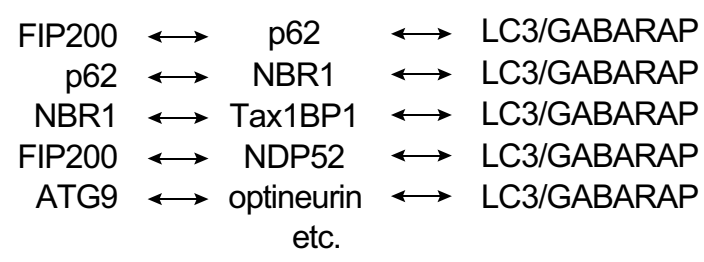

Isolation membrane/ phagophore

(B)
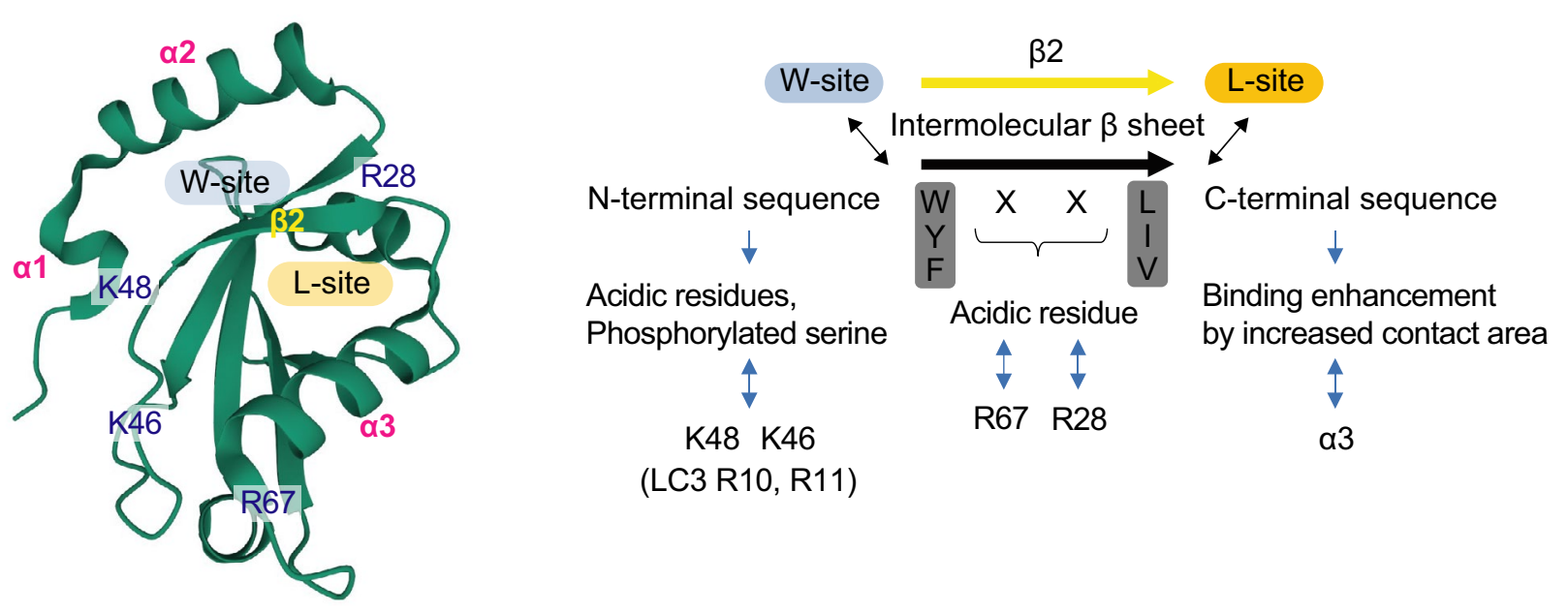

FIGURE 2 Receptors that facilitate selective autophagy by localizing cargo and binding to ubiquitin, and their interaction with ATG8 family proteins. A, Ubiquitin-binding (left) and cargo-localizing (right) receptors. B, Binding of AIM to ATG8. AIM adopts an elongated $\beta$ conformation and forms an intermolecular $\beta$-sheet with $\beta 2$ of ATG8. In addition, the W/Y/F-X-X-L/I/V side chain of the sequence binds to the $\mathrm{W}$-site between the $\mathrm{N}$-terminal helix $(\alpha 1, \alpha 2)$ and the ubiquitin fold, and the L/I/V side chain binds to the L-site between $\beta 2$ and $\alpha 3$ in the ubiquitin fold. AIMs often contain acidic or phosphorylated residues that act to enhance affinity by interacting electrostatically with conserved basic residues in ATG8 


\section{2 | Phase separation of cargo}

During the past $3 y$ in particular, researchers have made great strides in understanding selective autophagy. A conceptual diagram of the process is shown in Figure 3, using the selective autophagy of yeast aminopeptidase 1 (Ape1) $)^{2}$ and higher animal $p 62^{1}$ as examples. Selective autophagy, especially the degradation of soluble proteins, has been thought to involve a one-to-one degradation process in which ATG8 family proteins localized in isolation membranes/phagophores bind to their substrates. However, autophagosomes are large organelles, and therefore this method of degradation is extremely inefficient. This contrasts with the efficiency of the condensation of substrate molecules using liquid-liquid phase separation. ${ }^{29}$ To date, droplets such as the Ape1 complex, ${ }^{30}$ Ede1-dependent endocytic protein deposit (END), ${ }^{31}$ P-granules, ${ }^{32}$ stress granules, ${ }^{33}$ and p62-bodies ${ }^{34}$ have been found to be selectively degraded using autophagy. Furthermore, it has been found that several factors are necessary for selective degradation, specifically the retention of receptors on the surface of the phase-separated cargo, ${ }^{30}$ the appropriate degree of liquidity (gelation) of the droplets, ${ }^{16,30,32}$ and the wetting effect between the phase-separated cargo and the isolation membrane/phagophore. ${ }^{15}$ In addition, a model has been reported in which the formation of higher order assemblies of ER-phagy receptors in the local ER contributes to ER fragmentation and subsequent degradation. ${ }^{35,36}$

\section{3 | Interaction of receptors with upstream ATG proteins}

It has been thought that the binding of selective autophagy receptors to ATG8 family proteins is the decisive factor underlying the surrounding of specific cargos by the autophagosome. However, for yeast selective autophagy, the selective autophagy receptor binds not only to ATG8 but also to ATG11 (equivalent to FIP200 in higher animals). ${ }^{37}$ It is believed that ATG11 retains upstream ATG proteins on the cargo, causing it to be surrounded by newly formed isolation membranes/phagophores. ${ }^{37}$ Ubiquitin-binding receptors such as p62, NBR1, Tax1BP1, NDP52, and optineurin (core ubiquitin-binding receptors) bind to FIP200 through the FIP200-interacting region (FIR), ${ }^{11-13}$ and optineurin also binds to ATG9 $^{14}$ (Figure 2A). CCPG1 and TEX264, cargo-localizing receptors in ER-phagy, also bind to FIP200 through their FIR $^{38,39}$ (Figure 2A). In addition, core ubiquitinbinding receptors interact with each other, ${ }^{40-42}$ and the ULK1 protein kinase complex shows a differential ability to recruit them. ${ }^{43}$ The receptors on the cargo may work in a complementary manner to recruit upstream factors (eg, FIP200 and ATG9) and promote isolation membrane/phagophore formation. However, even when such receptors function in a complementary manner, there are cases in which essential receptors (NDP52 and optineurin) are present, such as in Parkin-PINK1-dependent mitophagy. ${ }^{44}$ Phosphorylation and dephosphorylation of each receptor are also involved in the regulation of interaction with ATG proteins. In particular, receptor phosphorylation, which has been identified in budding yeast, enhances binding to ATG11. ${ }^{37}$ In higher animals, phosphorylation of the ubiquitin-associated domain of p62 enhances its binding to ubiquitin chains, ${ }^{45,46}$ promoting liquid-liquid phase separation. Phosphorylation of FIR of p62 increases the binding ability to FIP200. ${ }^{11}$

\section{4 | ATG8 family protein}

Selective autophagy receptors directly interact with ATG8 family members (LC3A, LC3B, LC3C, GABARAP, GABARAPL1, and GABARAPL2). ${ }^{47}$ The amino acid motifs used for binding are named ATG8-family interacting motifs (AIMs) in yeast, LC3-interacting regions (LIRs) in LC3-bound types, and GABARAP-interaction motifs (GIMs) in GABARAP-bound types. ${ }^{47}$ Because the receptors interact with both the cargo and the ATG8 family-PE on the inner side of the isolation membrane/phagophore, the membrane is expected to elongate along the cargo. The core motifs of AIMs, LIRs, and GIMs consist of $[\mathrm{W} / \mathrm{F} / \mathrm{Y}]_{0}-\mathrm{X}_{1}-\mathrm{X}_{2}-[\mathrm{L} / \mathrm{V} / \mathrm{I}]_{3}$, where $[\mathrm{W} / \mathrm{F} / \mathrm{Y}]_{0}$ and $[\mathrm{L} / \mathrm{V} / \mathrm{I}]_{3}$ interact with 2 hydrophobic pockets: the gap between the $\mathrm{N}$-terminal $\alpha$-helix and the ubiquitin fold of the ATG 8 family and within the ubiquitin fold ${ }^{47}$ (Figure 2B). Autophagosome formation has also been observed in cells lacking ATG3, which is essential for ATG8 family$\mathrm{PE}$ conjugation, ${ }^{48}$ and autophagosomes surrounding mitochondria have been observed even in cells lacking all mammalian ATG8 family proteins. ${ }^{49}$ Conversely, when the interaction between ATG8 family proteins and p62-bodies on the isolation membrane/phagophore is inhibited, the number of autophagosomes surrounding p62-bodies decreases. ${ }^{15,16}$ In the absence of ATG8 family members, the receptors can recruit the ULK1 protein kinase complex and ATG9 to the vicinity of the cargo to form an isolation membrane/phagophore, which can randomly surround the cargo. In this way, it may be possible to surround the cargo randomly, albeit inefficiently.

\subsection{Client proteins}

The precursor of the yeast vacuolar enzyme Ape1, proApe1, forms liquid droplets (Ape1 complexes) by liquid-liquid phase separation. These droplets are surrounded by cytoplasm-to-vacuole targeting (Cvt) vesicles (equivalent to autophagosomes) and transported to the vacuole (Figure 3A). The Ape1 complex contains a group of proteins called clients that are not capable of droplet formation themselves, and these are transported to the vacuole together with the Ape1 complex. ${ }^{50}$ The transport of these clients to the vacuole has physiological effects such as the degradation of unwanted enzymes. ${ }^{50}$ In higher organisms, the regulation of cellular functions by selective autophagy is more complicated because of the presence of various signaling molecules that bind to receptor molecules as clients. For example, p62-bodies are thought to be liquid droplets formed in response to an increase in ubiquitinated proteins under stress (Figure 3B). The client Keap1 in such droplets 


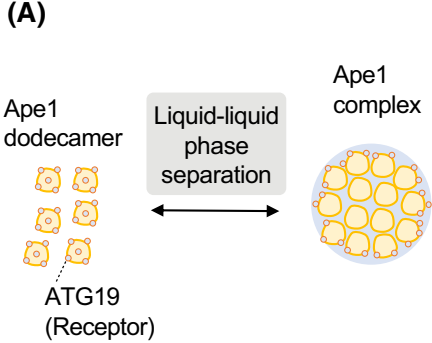

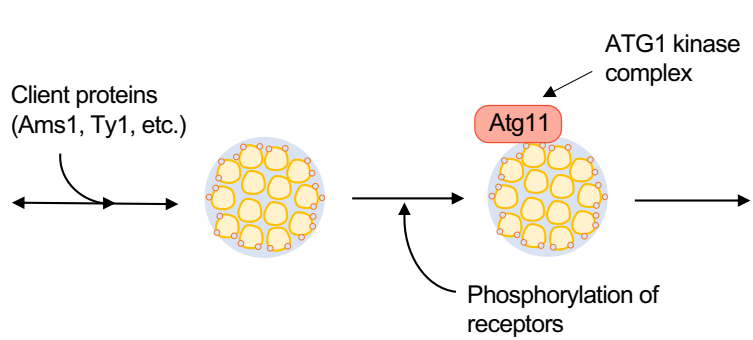

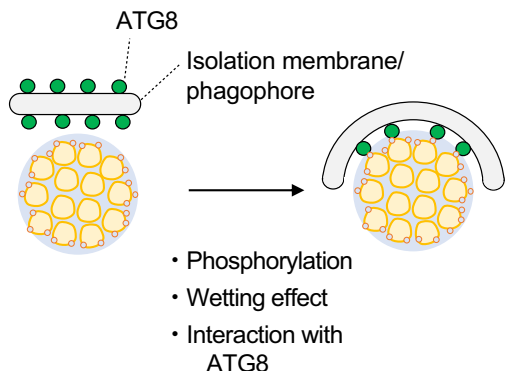

(B)
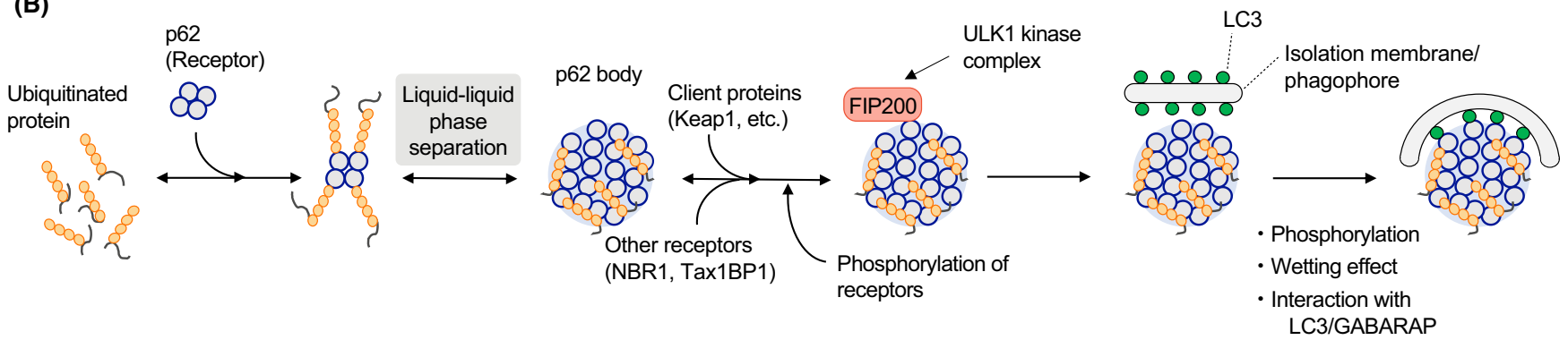

FIGURE 3 Schematic of the current understanding of the mechanism of selective autophagy. A, Selective autophagy of the Ape1 complex (Cvt pathway). proApe1 is condensed by liquid-liquid phase separation (forming the Ape1 complex). ATG19, a receptor protein localized on the surface of the Ape1 complex, recruits ATG11 and sequentially assembles ATG proteins to form an isolation membrane/ phagophore. The Ape1 complex is transported to the vacuole with client proteins such as Ams1. B, Selective autophagy of p62 bodies. The binding of $\mathrm{p} 62$ with ubiquitinated proteins causes liquid-liquid phase separation and then condensation (p62 bodies). p62 or the p62-binding protein NBR1 recruits FIP200, and isolation membranes/phagophores are formed by recruitment of ATG proteins. The p62 bodies are degraded in lysosomes with client proteins such as Keap1

is a ubiquitin ligase for the transcription factor Nrf2. When Keap1 is incorporated into the p62-body, Nrf2 escapes from the Keap1 interaction and translocates to the nucleus to induce the gene expression of Nrf2-target proteins, which function as antioxidants. ${ }^{51}$ When the K63 chains of ubiquitin are conjugated with intracellularly invading Salmonella typhimurium, the ubiquitin ligase complex LUBAC is recruited to generate linear polyubiquitin chains via the $\mathrm{N}$-terminal methionine of ubiquitin. ${ }^{27,28}$ The formation of linear ubiquitin chains by LUBAC promotes the transfer of the receptor optineurin and NEMO onto ubiquitinated murine typhus bacteria and induces the removal of bacteria by autophagy (xenophagy) and the activation of NF-kB, respectively, both of which inhibit bacterial growth. ${ }^{27,28}$

\section{3 | CONCLUDING REMARKS}

How far has our understanding of selective autophagy progressed? While breakthroughs have been made regarding the molecular mechanisms that drive the selective degradation of organelles by autophagy, there are still many unanswered questions about soluble proteins, including the following: (1) what proteins are selective substrates for autophagy, at what time points and in what cells and tissues, and what are the mechanisms involved? (2) What receptors promote autophagosome formation along these substrates? (3) What is the physiological significance of these substrates degradation? We are still far from having thorough answers to these questions. Regarding the first, comprehensive interactome analysis of core ubiquitin-binding receptors has been performed, ${ }^{52}$ and, in terms of the second, this same type of analysis has been used to investigate ATG8 family proteins. ${ }^{53}$ However, it is unknown how many substrates and receptors exist because these analyses have been conducted at the level of cultured cells under limited conditions.

In this review, we have mainly paid attention to the selectivity of macroautophagy, but additional mechanisms and substrates exist: microautophagy, for example, in which the lysosomal/ vacuolar membrane invaginates and takes in substrates, and membrane-permeable autophagy (including chaperone-mediated autophagy), in which substrates permeate the lysosomal membrane. In addition, it has been shown that endocytosis-mediated plasma membrane degradation (which is broadly defined as the lysosomal degradation of cellular components) and direct fusion autophagy (including crinophagy), both of which occur in specialized cells such as fertilized eggs in a time-specific manner, play important roles in individual development. All of these autophagy types are selective and contribute to the degradation of specific, spatiotemporally regulated proteins (including phase-separated granules and aggregates), nucleic acids, and organelles. Autophagy and its selective degradation of substrates are interrelated and may regulate a wide range of cellular functions such as environmental adaptation, homeostasis, and differentiation. 


\section{ACKNOWLEDGMENTS}

We would like to thank all Komatsu's laboratory members for helpful comments and suggestions. FMO is supported by a Japanese Government (Monbukagakusho: MEXT) Scholarship. YI is supported by a Grant-in-Aid for Scientific Research (C) (20K06644). MK is supported by a Grant-in-Aid for Scientific Research on Innovative Areas (19H05706), a Grant-in-Aid for Scientific Research (A) (21H004771), the Japan Society for the Promotion of Science (an A3 foresight program, to MK), and the Takeda Science Foundation.

\section{DISCLOSURE}

The authors have no conflict of interest.

\section{ORCID}

Mohammad Omar Faruk (iD https://orcid. org/0000-0001-7060-1870

Yoshinobu Ichimura iD https://orcid.org/0000-0003-0270-7766 Masaaki Komatsu iD https://orcid.org/0000-0001-7672-7722

\section{REFERENCES}

1. Bjørkøy G, Lamark T, Brech A, et al. p62/SQSTM1 forms protein aggregates degraded by autophagy and has a protective effect on huntingtin-induced cell death. J Cell Biol. 2005;171:603-614.

2. Lynch-Day MA, Klionsky DJ. The Cvt pathway as a model for selective autophagy. FEBS Lett. 2010;584:1359-1366.

3. Youle RJ, Narendra DP. Mechanisms of mitophagy. Nat Rev Mol Cell Biol. 2011;12:9-14.

4. Nakatogawa $\mathrm{H}$. Mechanisms governing autophagosome biogenesis. Nat Rev Mol Cell Biol. 2020;21:439-458.

5. Osawa T, Kotani T, Kawaoka T, et al. Atg2 mediates direct lipid transfer between membranes for autophagosome formation. Nat Struct Mol Biol. 2019;26:281-288.

6. Maeda S, Yamamoto H, Kinch LN, et al. Structure, lipid scrambling activity and role in autophagosome formation of ATG9A. Nat Struct Mol Biol. 2020;27:1194-1201.

7. Matoba K, Kotani T, Tsutsumi A, et al. Atg9 is a lipid scramblase that mediates autophagosomal membrane expansion. Nat Struct Mol Biol. 2020;27:1185-1193.

8. Huang D, Xu B, Liu LU, et al. TMEM41B acts as an ER scramblase required for lipoprotein biogenesis and lipid homeostasis. Cell Metab. 2021;33(8):1655-1670.e8.

9. Ghanbarpour A, Valverde DP, Melia TJ, Reinisch KM. A model for a partnership of lipid transfer proteins and scramblases in membrane expansion and organelle biogenesis. Proc Natl Acad Sci USA. 2021;118(16):e2101562118.

10. Lamark T, Johansen T. Mechanisms of selective autophagy. Annu Rev Cell Dev Biol. 2021;37(1). https://doi.org/10.1146/annurev-cellb io-120219-035530

11. Turco E, Witt M, Abert C, et al. FIP200 claw domain binding to p62 promotes autophagosome formation at ubiquitin condensates. Mol Cell. 2019;74(2):330-346.e11.

12. Vargas JNS, Wang C, Bunker E, et al. Spatiotemporal control of ULK1 activation by NDP52 and TBK1 during selective autophagy. Mol Cell. 2019;74(2):347-362.e6.

13. Ravenhill BJ, Boyle KB, von Muhlinen N, et al. The cargo receptor NDP52 initiates selective autophagy by recruiting the ULK complex to cytosol-invading bacteria. Mol Cell. 2019;74(2):320-329.e6.

14. Yamano K, Kikuchi R, Kojima W, et al. Critical role of mitochondrial ubiquitination and the OPTN-ATG9A axis in mitophagy. J Cell Biol. 2020;219(9):e201912144.
15. Agudo-Canalejo J, Schultz SW, Chino H, et al. Wetting regulates autophagy of phase-separated compartments and the cytosol. Nature. 2021;591:142-146.

16. Kageyama S, Gudmundsson SR, Sou Y-S, et al. p62/SQSTM1droplet serves as a platform for autophagosome formation and anti-oxidative stress response. Nat Commun. 2021;12:16.

17. Khaminets A, Behl C, Dikic I. Ubiquitin-dependent and independent signals in selective autophagy. Trends Cell Biol. 2016;26:6-16.

18. Wilkinson S. ER-phagy: shaping up and destressing the endoplasmic reticulum. FEBS J. 2019;286:2645-2663.

19. Chino H, Mizushima N. ER-Phagy: quality control and turnover of endoplasmic reticulum. Trends Cell Biol. 2020;30:384-398.

20. Onishi M, Yamano K, Sato M, Matsuda N, Okamoto K. Molecular mechanisms and physiological functions of mitophagy. EMBO J. 2021;40:e104705.

21. Pickrell AM, Youle RJ. The roles of PINK1, parkin, and mitochondrial fidelity in Parkinson's disease. Neuron. 2015;85:257-273.

22. Nguyen TN, Padman BS, Lazarou M. Deciphering the molecular signals of PINK1/Parkin mitophagy. Trends Cell Biol. 2016;26:733-744.

23. Chauhan S, Kumar S, Jain A, et al. TRIMs and galectins globally cooperate and TRIM16 and Galectin-3 co-direct autophagy in endomembrane damage homeostasis. Dev Cell. 2016;39:13-27.

24. Yoshida Y, Yasuda S, Fujita T, et al. Ubiquitination of exposed glycoproteins by SCF(FBXO27) directs damaged lysosomes for autophagy. Proc Natl Acad Sci USA. 2017;114:8574-8579.

25. Koerver L, Papadopoulos C, Liu B, et al. The ubiquitin-conjugating enzyme UBE2QL1 coordinates lysophagy in response to endolysosomal damage. EMBO Rep. 2019;20:e48014.

26. Otten EG, Werner E, Crespillo-Casado A, et al. Ubiquitylation of lipopolysaccharide by RNF213 during bacterial infection. Nature. 2021;594(7861):111-116.

27. Noad J, von der Malsburg A, Pathe C, Michel MA, Komander D, Randow F. LUBAC-synthesized linear ubiquitin chains restrict cytosol-invading bacteria by activating autophagy and NF-kappaB. Nat Microbiol. 2017;2:17063.

28. van Wijk SJL, Fricke F, Herhaus L, et al. Linear ubiquitination of cytosolic Salmonella Typhimurium activates NF-kappaB and restricts bacterial proliferation. Nat Microbiol. 2017;2:17066.

29. Noda NN, Wang Z, Zhang H. Liquid-liquid phase separation in autophagy. J Cell Biol. 2020;219(8):e202004062.

30. Yamasaki A, Alam JM, Noshiro D, et al. Liquidity is a critical determinant for selective autophagy of protein condensates. Mol Cell. 2020;77(6):1163-1175.e9.

31. Wilfling F, Lee C-W, Erdmann PS, et al. A selective autophagy pathway for phase-separated endocytic protein deposits. Mol Cell. 2020;80(5):764-778.e7.

32. Zhang G, Wang Z, Du Z, Zhang H. mTOR regulates phase separation of PGL granules to modulate their autophagic degradation. Cell. 2018;174(6):1492-1506.e22.

33. Turakhiya A, Meyer SR, Marincola G, et al. ZFAND1 recruits p97 and the $26 \mathrm{~S}$ proteasome to promote the clearance of arseniteinduced stress granules. Mol Cell. 2018;70(5):906-919.e7.

34. Sun D, Wu R, Zheng J, Li P, Yu L. Polyubiquitin chain-induced p62 phase separation drives autophagic cargo segregation. Cell Res. 2018;28:405-415

35. Bhaskara RM, Grumati P, Garcia-Pardo J, et al. Curvature induction and membrane remodeling by FAM134B reticulon homology domain assist selective ER-phagy. Nat Commun. 2019;10:2370.

36. Mochida K, Yamasaki A, Matoba K, Kirisako H, Noda NN, Nakatogawa H. Super-assembly of ER-phagy receptor Atg40 induces local ER remodeling at contacts with forming autophagosomal membranes. Nat Commun. 2020;11:3306.

37. Farre JC, Subramani S. Mechanistic insights into selective autophagy pathways: lessons from yeast. Nat Rev Mol Cell Biol. 2016;17:537-552. 
38. Smith MD, Harley ME, Kemp AJ, et al. CCPG1 is a non-canonical autophagy cargo receptor essential for ER-phagy and pancreatic ER proteostasis. Dev Cell. 2018;44(2):217-232.e11.

39. Chino H, Hatta T, Natsume T, Mizushima N. Intrinsically disordered protein TEX264 mediates ER-phagy. Mol Cell. 2019;74(5):909-921. e6.

40. Ohnstad AE, Delgado JM, North BJ, et al. Receptor-mediated clustering of FIP200 bypasses the role of LC3 lipidation in autophagy. EMBO J. 2020;39:e104948.

41. Zaffagnini G, Savova A, Danieli A, et al. p62 filaments capture and present ubiquitinated cargos for autophagy. EMBO J. 2018;37(5):e98308.

42. Sanchez-Martin P, Sou YS, Kageyama S, Koike M, Waguri S, Komatsu M. NBR1-mediated p62-liquid droplets enhance the Keap1-Nrf2 system. EMBO Rep. 2020;21:e48902.

43. Chang $C$, Shi X, Jensen LE, et al. Reconstitution of cargo-induced LC3 lipidation in mammalian selective autophagy. Sci Adv. 2021;7(17):eabg4922.

44. Lazarou M, Sliter DA, Kane LA, et al. The ubiquitin kinase PINK1 recruits autophagy receptors to induce mitophagy. Nature. 2015;524:309-314

45. Matsumoto G, Wada K, Okuno M, Kurosawa M, Nukina N. Serine 403 phosphorylation of p62/SQSTM1 regulates selective autophagic clearance of ubiquitinated proteins. Mol Cell. 2011;44:279-289.

46. Pilli M, Arko-Mensah J, Ponpuak M, et al. TBK-1 promotes autophagy-mediated antimicrobial defense by controlling autophagosome maturation. Immunity. 2012;37:223-234.
47. Kirkin V, Rogov VV. A diversity of selective autophagy receptors determines the specificity of the autophagy pathway. Mol Cell. 2019;76:268-285.

48. Tsuboyama K, Koyama-Honda I, Sakamaki Y, Koike M, Morishita $\mathrm{H}$, Mizushima N. The ATG conjugation systems are important for degradation of the inner autophagosomal membrane. Science. 2016;354:1036-1041.

49. Nguyen TN, Padman BS, Usher J, Oorschot V, Ramm G, Lazarou M. Atg8 family LC3/GABARAP proteins are crucial for autophagosomeIysosome fusion but not autophagosome formation during PINK1/ Parkin mitophagy and starvation. J Cell Biol. 2016;215:857-874.

50. Yamasaki A, Noda NN. Structural biology of the Cvt pathway. J Mol Biol. 2017;429:531-542

51. Sanchez-Martin P, Komatsu M. p62/SQSTM1 - steering the cell through health and disease. J Cell Sci. 2018;131(21):jcs222836.

52. Zellner S, Schifferer M, Behrends C. Systematically defining selective autophagy receptor-specific cargo using autophagosome content profiling. Mol Cell. 2021;81(6):1337-1354.e8.

53. Behrends C, Sowa ME, Gygi SP, Harper JW. Network organization of the human autophagy system. Nature. 2010;466:68-76.

How to cite this article: Faruk MO, Ichimura Y, Komatsu M. Selective autophagy. Cancer Sci. 2021;112:3972-3978. https:// doi.org/10.1111/cas.15112 